\title{
Excess white noise to probe transport mechanisms in a membrane channel
}

\author{
María Queralt-Martín, ${ }^{*}$ M. Lidón López, and Antonio Alcaraz \\ Laboratory of Molecular Biophysics, Department of Physics, Universitat Jaume I, 12071 Castellón, Spain
}

(Received 23 March 2015; published 8 June 2015)

\begin{abstract}
Current fluctuation analysis has been successfully used over the years to investigate the physical properties of different systems. Here, we perform single-channel time-resolved current experiments in a protein channel to evaluate the different transport mechanisms governing the channel function. Using different salts of monovalent and divalent cations in a wide range of concentrations and applied potentials, we analyze current fluctuations focusing on the voltage dependence of the additional white noise that appears in the low-frequency range of the spectra. We demonstrate that the channel displays two characteristic transport regimes: at low salt concentrations $(10 \mathrm{mM}$ to $1 \mathrm{M})$ ion permeation is controlled by the protein fixed charges that induce accumulation or exclusion of ions to preserve local electroneutrality. At high salt concentrations $(>1 \mathrm{M})$ adsorption processes associated to the binding of cations to the channel charges regulate the transport properties.
\end{abstract}

DOI: 10.1103/PhysRevE.91.062704

PACS number(s): 87.16.Vy, 66.10.Ed, 72.70.+m

\section{INTRODUCTION}

The analysis of fluctuations provides a rich source of information about the physical mechanisms responsible for the generation of noise. Different types of noise have been analyzed over recent years to describe successfully the properties of a wide variety of phenomena as shot noise in lattice gases [1] and electrical conductors [2] or $1 / f$ noise in biological membranes [3]. In addition, noise analysis has been successfully employed in protein ion channels to investigate the transport mechanisms that control the channel function [4-7]. In this work we study current fluctuations to assess the different transport mechanisms occurring in the bacterial porin $\mathrm{OmpF}$, a wide and weakly selective channel in the outer membrane of Escherichia coli [8]. The high-resolution 3D structure of OmpF reveals a homotrimer with each monomer forming a 16-stranded $\beta$-barrel that spans the lipid bilayer and serves as a water-filled, voltage-gated pore [9] with multiionic transport. Although considered a wide channel, OmpF porin is small enough so that the pore residues do have a decisive influence on the channel permeability $[8,10-14]$. Noise analysis has been previously used in OmpF to investigate the $\mathrm{pH}$ titration of the channel residues [4]. It was shown that the power spectral density could be represented by a sum of Lorentzians plus the background spectrum, emphasizing also that in a wide range of $\mathrm{pH}$ (from $\mathrm{pH} 1$ to $\mathrm{pH} 12$ ), the OmpF channel noise was free from $1 / f$ fluctuations. Here we follow a complementary approach based on the noise studies of Hoogerheide et al. in synthetic nanopores [15]. We pay attention to the appearance of an additional white noise in the low-frequency range. The average noise scales with the square of the dc current, showing that this excess frequencyindependent noise derives from conductance fluctuations. We analyze how these fluctuations depend on ionic concentration in different electrolytes $\left(\mathrm{KCl}, \mathrm{LiCl}, \mathrm{MgCl}_{2}\right.$, and $\left.\mathrm{CaCl}_{2}\right)$ to disclose the different transport mechanisms occurring in $\mathrm{OmpF}$ channel. Our results emphasize that noise analysis can be used as a sensitive tool to study protein channel dynamics and chemistry.

\footnotetext{
*mqueralt@uji.es
}

\section{MATERIAL AND METHODS}

We analyze the current fluctuations from single-channel measurements performed using the V-clamp technique. The planar lipid membrane was created in a two-compartment (cis and trans) Teflon chamber using 1,2-diphytanoyl-snglycero-3-phosphocholine (DPhPC) lipid by the apposition of monolayers across orifices with diameters of $70-100 \mu \mathrm{m}$ on a $15-\mu \mathrm{m}$-thick Teflon partition. The orifices were pretreated with a $1 \%$ solution of hexadecane in pentane. The electrolyte (monovalent or divalent) solution was adjusted at $\mathrm{pH} 6$ and controlled during the experiments using a GLP22 $\mathrm{pH}$ meter (Crison). The bacterial porin OmpF, kindly provided by Dr. S. M. Bezrukov (NIH, Bethesda, MD), was added at the cis side of the chamber. Single-channel current recordings were obtained using an Axopatch 200B amplifier (Molecular Devices, Sunnyvale, CA) with an inline low-pass Bessel filter at $10 \mathrm{kHz}$. Data were digitalized with a sampling frequency of $50 \mathrm{kHz}$ using a Digidata 1440 (Molecular Devices, Sunnyvale, $\mathrm{CA}$ ). The chamber was isolated from external noise sources with a double metal screen (Amuneal Manufacturing Corp., Philadelphia, PA). An electric potential was applied using $\mathrm{Ag} / \mathrm{AgCl}$ electrodes in $2 \mathrm{M} \mathrm{KCl} ; 1.5 \%$ agarose bridges were assembled within standard 250-ml pipette tips. The potential was defined as positive when it was higher on the side of the protein addition.

\section{RESULTS AND DISCUSSION}

Figure 1(a) shows a representative high-resolution current trace obtained from single-open-channel recordings. The average current depends on the concentration and type of electrolyte. As could be expected, higher currents are obtained with higher salt concentrations, both for salts of monovalent and divalent cations. Interestingly, the recordings show fast current fluctuations, with very short elementary events, as shown at a finer time scale in the inset on the right. To characterize the origin of these fast events that appear at all the conditions explored-wide range of concentrations and applied potentials in salts of monovalent $(\mathrm{KCl}$ and $\mathrm{LiCl})$ and divalent $\left(\mathrm{MgCl}_{2}\right.$ and $\left.\mathrm{CaCl}_{2}\right)$ cations-we calculated the power spectral densities (PSDs) from the current fluctuations of each 
(a)

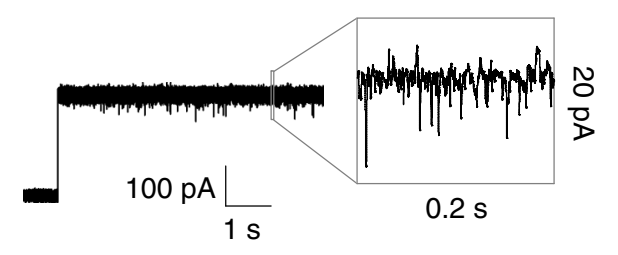

(b)
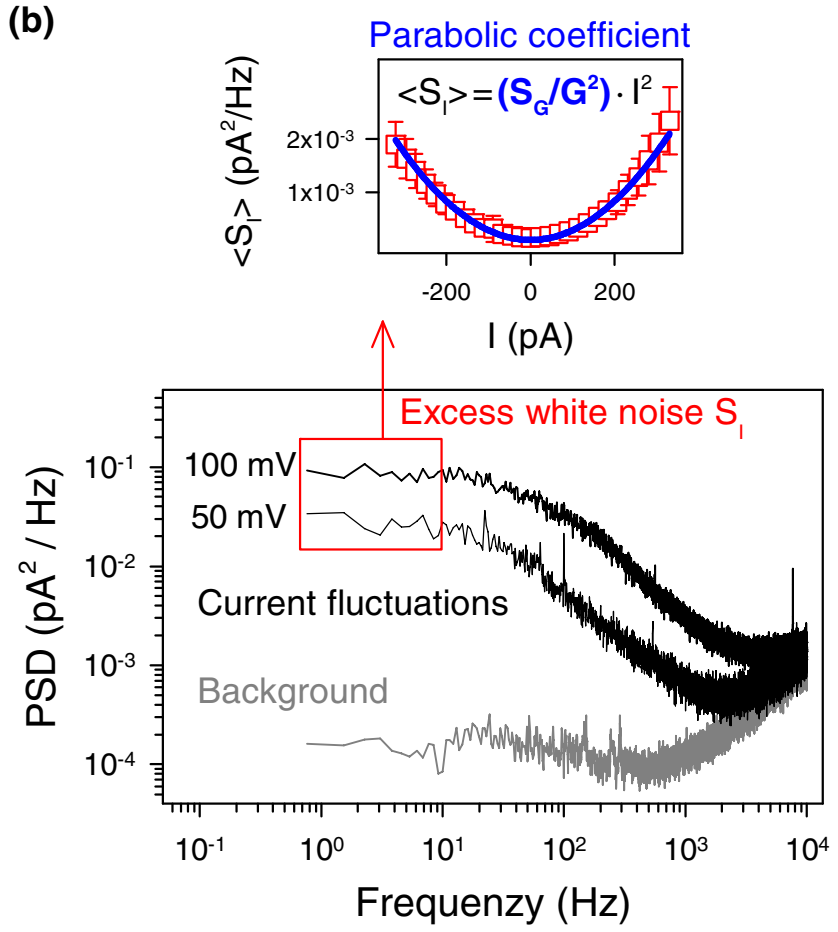

FIG. 1. (Color online) (a) Representative single-channel trace recorded at $100 \mathrm{mV}$ of applied potential. The inset shows the current recording at a finer time scale after the application of an 8-pole Bessel filter with $500 \mathrm{~Hz}$ cutoff frequency. (b) Typical power spectral density (PSD) at 50 and $100 \mathrm{mV}$ compared to the background spectra (recorded at $0 \mathrm{mV}$ ). The averaged excess white noise of the current fluctuations $(0-20 \mathrm{~Hz}$ band) is evaluated as a function of the applied voltage (open squares) to obtain the parabolic coefficient $S_{G} / G^{2}$ from a parabolic fit (solid line). The error bars correspond to the deviation of the mean calculated from the low-frequency range of the spectra. The experiments shown were performed at $2 \mathrm{M} \mathrm{MgCl}_{2}$, pH 6.

experiment. Figure 1(b) (lower part) shows a typical PSD for +50 and $+100 \mathrm{mV}$ applied voltages in comparison to the spectrum of the background noise. The background noise was measured at zero applied voltage.

The PSD provides a rich source of information about the underlying physical mechanisms that generate current fluctuations. Because each source of noise generates a characteristic pattern in the PSD, the analysis of current fluctuations allows extracting kinetic information about physical mechanisms that many times is difficult to obtain directly from the current measurements [16].

As shown in previous studies [4], the PSD calculated from the current flowing through the OmpF channel shows a characteristic Lorentzian-like shape: a white noise dominates the spectra at low frequencies (up to $50 \mathrm{~Hz}$ ), with a constant PSD. In spite of this characteristic shape, a single Lorentzian is actually not able to fit the PSD but a sum of a minimum of two is necessary to adequately represent the experimental data [4]. Because this complicates the interpretation of the physical constants obtained, we decided to follow a complementary approach.

To probe the current fluctuations associated to each experimental condition, we focused on the excess white noise emerging in the low-frequency region of the spectra. The average level of this constant region, $\left\langle S_{I}\right\rangle$, changes with the applied voltage bias so that higher potentials yield higher values of the white noise. In the case that $\left\langle S_{I}\right\rangle$ increases as $V^{2}$, then the origin of the fast events recorded in the current traces can be related to the existence of conductance fluctuations [15]. This is what we actually observe, as shown in the upper part of Fig. 1(b): the averaged low-frequency PSD is proportional to $I^{2}$, which is proportional to $V^{2}$. The relation between $\left\langle S_{I}\right\rangle$ and the conductance fluctuations, $S_{G}$, is obtained through the equation $\left\langle S_{I}\right\rangle=\left(S_{G} / G^{2}\right) I^{2}$. The parabolic coefficient $S_{G} / G^{2}$ contains relevant physicochemical information of the intrinsic system fluctuations. In particular, it has been related to the number of particles that fluctuates at the pore walls [15]. Consequently, we performed a parabolic fit of the averaged low-frequency noise $\left\langle S_{I}\right\rangle$ plotted versus the current $I$ to obtain the parabolic coefficient $S_{G} / G^{2}$ from each experiment.

The rationalization of the parabolic coefficients requires first an assessment of the channel transport mechanisms involved in the channel conductance. Current-voltage $(I-V)$ relationships were always linear (data not shown), so that the voltage-independent channel conductance can be calculated simply as $G=I / V$. Figure 2 displays the channel conductance versus electrolyte activity. The use of electrolyte activity instead of concentration is necessary to separate the role of the channel from the intrinsic properties of the electrolyte that become important for salts of divalent cations at high molarity [17,18]. Figure 2 demonstrates that the channel conductance measured in salts of monovalent and divalent cations displays common trends. This is consistent with the lack of specificity of this wide channel reported in previous studies [4,8]. In all electrolytes under study the conductance scales as $G \sim a^{3 / 4}$ for low activities. In the high-activity regime we find saturation or even a slight decrease in conductance that scales as $G \sim a^{-1 / 4}$. In the case of $\mathrm{KCl}$ this late regime is not attained because of the solubility limit.

Because there are no external activity gradients in the system, the channel conductance should be closely proportional to the local conductivity [19]. Therefore, we can write the conductance as a function of the ion activities $a_{i}$, the ion diffusion coefficients $D_{i}$, and their valences $z_{i}$ [18]:

$$
G \sim z_{+}^{2} D_{+} a_{+}+z_{-}^{2} D_{-} a_{-} .
$$

The channel charges induce the accumulation of counterions and the exclusion of coions to preserve local electroneutrality. The Donnan equilibrium formalism [19,20] provides the theoretical framework to account for the effective ion activities inside the pore $a_{+}$and $a_{-}$, which depend on the channel fixed-charge concentration $X$. For the case of monovalent salts, 

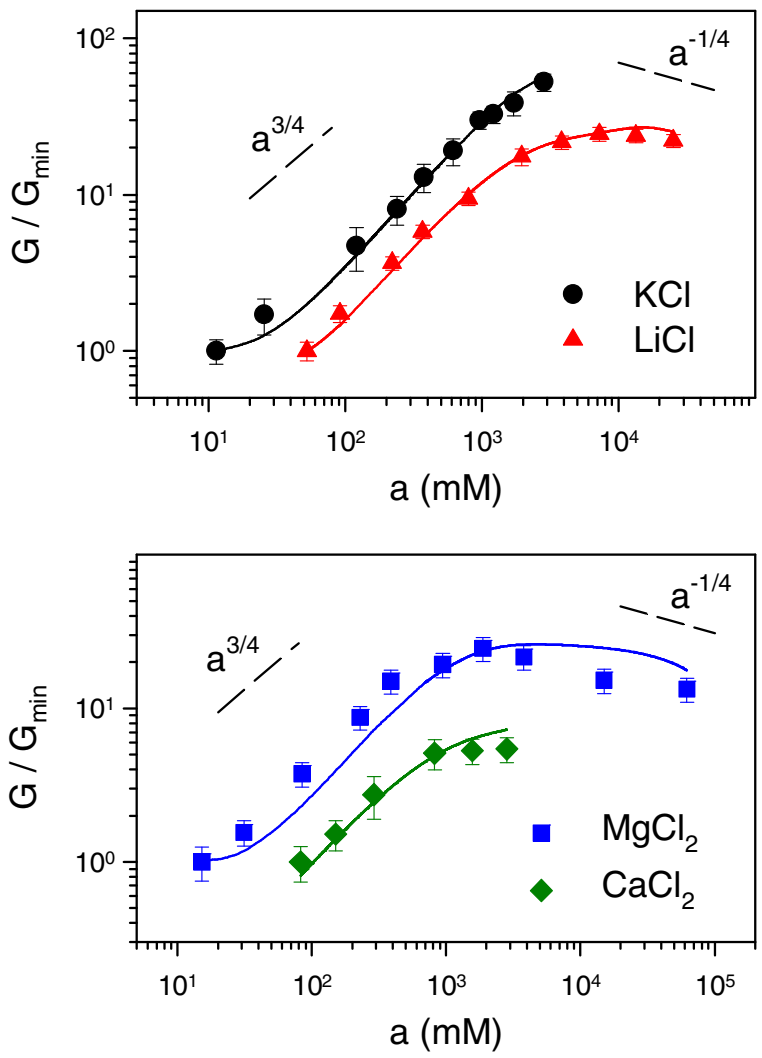

FIG. 2. (Color online) Normalized OmpF channel conductance versus electrolyte activity in monovalent (upper panel) and divalent (lower panel) salts. The dashed lines show the qualitative trends observed. The solid lines are calculated applying a modified Donnan formalism as described in the main text. All the experiments were performed at $\mathrm{pH} 6$.

they are described with the following equations:

$$
\begin{aligned}
& a_{+}=-\frac{X}{2}+\sqrt{\left(\frac{X}{2}\right)^{2}+a^{2},} \\
& a_{-}=\frac{X}{2}+\sqrt{\left(\frac{X}{2}\right)^{2}+a^{2},}
\end{aligned}
$$

where $a$ is the electrolyte bulk activity. Equation (2) sets two limiting trends for the conductance as a function of activity: when $a \ll X$, local ion activities are just proportional to the channel fixed charge concentration in order to preserve charge neutrality. This means that in this regime both $a_{+}$and $a_{-}$are independent of the bulk activities and so is the conductance. When $a \gg X$, the Donnan exclusion is salted out and ion activities inside the channel are equal to bulk activities and hence $G \sim a^{1}$. The experiments reported in Fig. 2 show that the modulation of the experimental $G$ is halfway between the two limits, yielding $G \sim a^{3 / 4}$. This indicates that in the experiments performed the mean ion activities are comparable to the fixed charge concentration of the channel, and the limiting cases mentioned before are not clearly attained. In the case of divalent cations the Donnan equations cannot be solved analytically, but numerical calculations give a similar trend [18].
To account for the saturation or even decrease of conductance observed at high activities in Fig. 2, we included two additional factors into the model. First, the dependence of diffusion coefficients on ion activity, calculated though the relation between the measured bulk ion conductivity and the ion activity, for each electrolyte [18]. This dependence explains why the conductance in $\mathrm{LiCl}$ is quite different from that of $\mathrm{KCl}$ or the small differences found between $\mathrm{CaCl}_{2}$ and $\mathrm{MgCl}_{2}$ [17]. Second, the variation in the concentration of mobile ions inside the channel due to adsorption processes as suggested by a number of different approaches. Thus, fluorescence experiments [21], MD simulations [22], free-energy calculations [13], and site-directed mutagenesis experiments [23] pointed to the existence of a binding site for monovalent cations located around the central constriction of the OmpF channel $[13,14,22]$. X-ray structures in $\mathrm{MgCl}_{2}$ showed a binding site in the selectivity filter [24] as confirmed by selectivity experiments involving selected mutants in salts of multivalent cations [25]. In spite of all this information, the functional role of that binding site remained unclear since no blocking events have been observed and the channel displays Ohmic conduction in all conditions studied [25]. This is probably because $\mathrm{OmpF}$ is a wide channel, and the cation binding does not occlude the narrower part of the pore but just modifies the number of mobile counterions via an adsorption process (the cations bound to the binding site do not contribute to the channel current). This effect can be included in the calculations using a Langmuir adsorption isotherm [19] with the corresponding binding constant $K_{d}$ as a free parameter.

Thus, after introducing the two additional factors, the equation describing the conductance in the modified Donnan formalism reads as follows:

$$
\begin{aligned}
G & \sim z_{+}^{2} D_{+}(a) a_{\mathrm{eff}+}+z_{-}^{2} D_{-}(a) a_{-} \\
& =z_{+}^{2} D_{+}(a) \frac{a_{+}}{1+a / K_{d}}+z_{-}^{2} D_{-}(a) a_{-},
\end{aligned}
$$

Despite the simplicity of this approach, the model reproduces the two trends observed in the experiments. The solid lines shown in Fig. 2 correspond to the fit of the experimental data to Eq. (3). The fitting parameters obtained, $X=$ $-55 \mathrm{mM}(\mathrm{KCl}), X=-120 \mathrm{mM}(\mathrm{LiCl}), X=60 \mathrm{mM}\left(\mathrm{MgCl}_{2}\right.$ and $\left.\mathrm{CaCl}_{2}\right)$ and $K_{d}=80 \mathrm{M}(\mathrm{KCl}$ and $\mathrm{LiCl}), K_{d}=70 \mathrm{M}$ $\left(\mathrm{MgCl}_{2}\right.$ and $\left.\mathrm{CaCl}_{2}\right)$, are of the order of magnitude expected.

In any case, the qualitative message is clear: the fixed charge of the pore exerts a control of the ion transport and this control is enough to explain the behavior of conductance at the low-activity regime, regardless of the type of ions present in the system. In addition, the existence of a binding site for cations, altogether with the solution effects, explains the trend at high activities.

Next, we analyzed the current fluctuations over different solution activities to identify the transport mechanisms that regulate the channel conductance. The conductance fluctuation $S_{G} / G^{2}$ is determined from the parabolic fit of the lowfrequency excess white noise versus current. Figure 3 shows the results obtained as a function of salt activity. For all electrolytes under study, the parabolic coefficient exhibits two different regimes, $S_{G} / G^{2} \sim a^{-3 / 2}$ at low activities and $S_{G} / G^{2} \sim a^{3 / 2}$ at high activities. Interestingly, the inflection 

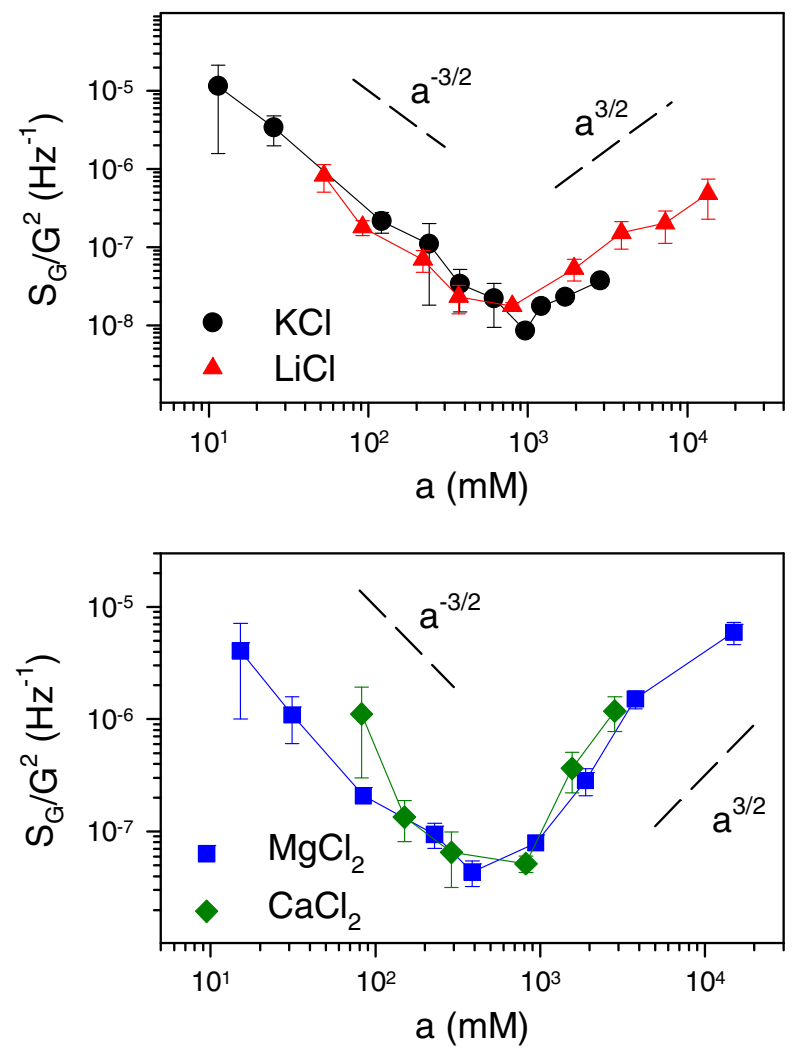

FIG. 3. (Color online) Dependence of the parabolic coefficient $S_{G} / G^{2}$ on electrolyte activity, for monovalent (upper panel) and divalent (lower panel) salts. The dashed lines show the two regimes observed. The solid lines are drawn to guide the eye. All the experiments were performed at $\mathrm{pH} 6$.

point is observed around $a \sim 1 \mathrm{M}$, similar to the onset of conductance saturation in Fig. 2.

The number of particles that contribute to the conductance noise, $S_{G}$, can be calculated combining the conductance measurements from Fig. 2 with the parabolic coefficient shown in Fig. 3, as follows:

$$
S_{G}=G^{2} \frac{S_{G}}{G^{2}},
$$

The results obtained from Eq. (4) are shown in Fig. 4.

Thus, the conductance fluctuations at the low activity regime yield $S_{G} \sim a^{0}$. This means that the number of fluctuating particles remains constant when increasing the activity. Having in mind that only for surface-bound fluctuations does the parabolic coefficient $S_{G} / G^{2}$ scale as $a^{-3 / 2}$ [15], the fluctuating particles could be just the counterions screening the channel charges located in the pore surface as explained by Donnan equilibrium. This result is consistent with a transport regime in which the electroneutrality requirements arising from the channel fixed charge dictate the channel conductance.

In the case of high activities, $S_{G} \sim a^{1}$. This result cannot be ascribed to volume fluctuations (as those suggested by the Hooge relation) or geometric fluctuations due to an openingclosing of the pore, because they would both induce $1 / f$ PSDs $[6,26]$, which are not observed in our results, as shown in Fig. 1.

Interestingly, $S_{G} \sim a^{1}$ can be understood invoking again the binding processes that dominate the channel conductance in
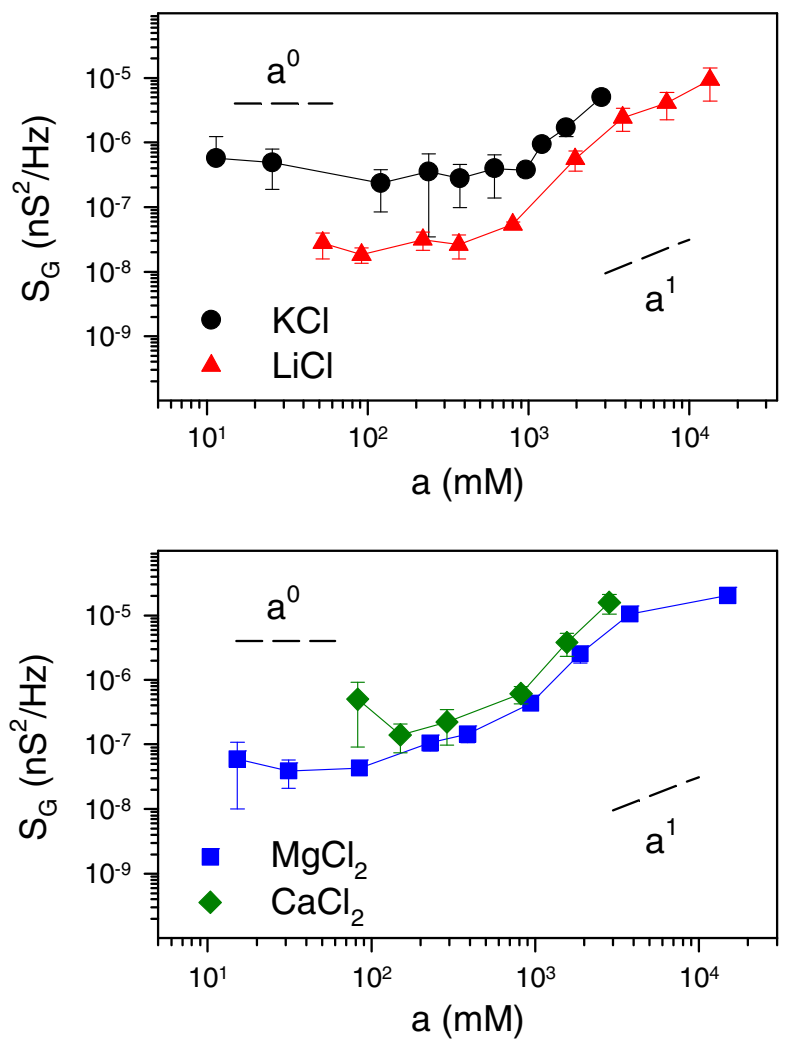

FIG. 4. (Color online) Dependence of $S_{G}$ on electrolyte activity, for monovalent (upper panel) and divalent (lower panel) salts. The dashed lines show the two regimes observed. The solid lines are drawn to guide the eye. All the experiments were performed at $\mathrm{pH} 6$.

the high-activity regime as shown in Fig. 2. We can assume that the fraction of occupied sites is directly related to the quantity of fluctuating particles that contributes to the noise. Using the Langmuir adsorption isotherm [19] to account for that binding, the fraction of occupied sites varies linearly with solution activity when the activity of cations is lower than the effective dissociation constant $K_{d}$. Accordingly, the conductance fluctuations are expected to follow $S_{G} \sim a^{1}$, which is what we actually observe in Fig. 4. This explanation is consistent with the high $K_{d}$ values obtained in the fitting of Fig. 2 .

Therefore, the study of current fluctuations provides direct functional evidence of the transport mechanisms occurring in the protein channel showing features that may appear undetected in the current traces. This is especially evident at high salt concentration. In contrast to conductance experiments, noise analysis shows a well-defined common trend clearly visible in all electrolytes under study. The adsorption processes related to the binding site for cations that are hardly visible in conductance experiments for $\mathrm{KCl}$ or $\mathrm{CaCl}_{2}$ can be detected and elucidated using current fluctuation analysis.

\section{CONCLUSIONS}

By means of a particular current fluctuation analysis technique combined with conductance experiments we have demonstrated the existence of two main ion transport regimes in the OmpF channel, both for salts of monovalent and divalent cations. At low salt concentrations, the electroneutrality 
requirements arising from the pore-fixed charges determine the channel current. At high salt concentrations, the experimental results are consistent with the existence of a binding site for cations that governs the transport properties, providing a key functional evidence of this binding in salts of monovalent ions. Our results also emphasize the importance of noise analysis as a tool to study protein channel electrochemistry.

\section{ACKNOWLEDGMENTS}

We acknowledge Dr. David P. Hoogerheide for his critical reading of the manuscript and his help with the noise analysis. We also acknowledge the support from the Spanish Ministry of Economy and Competitiveness (MINECO Project No. FIS2013-40473-P) and the Fundació Caixa Castelló-Bancaixa (Project No. P1-1B2012-03).
[1] B. Meerson and P. V. Sasorov, Phys. Rev. E 89, 010101(R) (2014).

[2] Y. M. Blanter and M. Buttiker, Phys. Rep. 336, 1 (2000).

[3] S. M. Bezrukov and M. Winterhalter, Phys. Rev. Lett. 85, 202 (2000).

[4] E. M. Nestorovich, T. K. Rostovtseva, and S. M. Bezrukov, Biophys. J. 85, 3718 (2003).

[5] P. A. Gurnev, D. Harries, V. A. Parsegian, and S. M. Bezrukov, J. Phys.: Condens. Matter 22, 454110 (2010).

[6] Z. S. Siwy and A. Fuliński, Phys. Rev. Lett. 89, 158101 (2002).

[7] C. Hilty and M. Winterhalter, Phys. Rev. Lett. 86, 5624 (2001).

[8] H. Nikaido, Microbiol. Mol. Biol. Rev. 67, 593 (2003).

[9] S. W. Cowan, T. Schirmer, G. Rummel, M. Steiert, R. Ghosh, R. A. Pauptit, J. N. Jansonius, and J. P. Rosenbusch, Nature 358, 727 (1992).

[10] R. Benz, A. Schmid, and R. E. Hancock, J. Bacteriol. 162, 722 (1985).

[11] A. H. Delcour, Front. Biosci. 8, D1055 (2003).

[12] A. Alcaraz, E. M. Nestorovich, M. Aguilella-Arzo, V. M. Aguilella, and S. M. Bezrukov, Biophys. J. 87, 943 (2004).

[13] C. Danelon, A. Suenaga, M. Winterhalter, and I. Yamato, Biophys. Chem. 104, 591 (2003).
[14] W. Im and B. Roux, J. Mol. Biol. 319, 1177 (2002).

[15] D. P. Hoogerheide, S. Garaj, and J. A. Golovchenko, Phys. Rev. Lett. 102, 256804 (2009).

[16] S. M. Bezrukov, Fluct. Noise Lett. 4, L23 (2004).

[17] R. A. Robinson and R. H. Stokes, Electrolyte Solutions: Second Revised Edition, 2nd ed. (Dover Publications, London, 2002).

[18] E. García-Giménez, M. L. López, V. M. Aguilella, and A. Alcaraz, Biochem. Biophys. Res. Commun. 404, 330 (2011).

[19] B. Hille, Ion Channels of Excitable Membranes, 3rd ed. (Sinauer Associates Inc., Sunderland, MA, 2001).

[20] N. Lakshminarayanaiah, Transport Phenomena in Membranes (Academic Press, New York, 1969).

[21] Y. Kobayashi and T. Nakae, Eur. J. Biochem. 151, 231 (1985).

[22] A. Suenaga, Y. Komeiji, M. Uebayasi, T. Meguro, M. Saito, and I. Yamato, Biosci. Rep. 18, 39 (1998).

[23] A. Alcaraz, M. Queralt-Martín, E. García-Giménez, and V. M. Aguilella, Biochim. Biophys. Acta-Biomembranes 1818, 2777 (2012).

[24] E. Yamashita, M. V Zhalnina, S. D. Zakharov, O. Sharma, and W. A. Cramer, EMBO J. 27, 2171 (2008).

[25] A. Alcaraz, E. M. Nestorovich, M. L. López, E. García-Giménez, S. M. Bezrukov, and V. M. Aguilella, Biophys. J. 96, 56 (2009).

[26] F. N. Hooge, Phys. Lett. A 29, 139 (1969). 